\title{
An Algorithm for Coarse Particle Sedimentation Simulation by Stokesian Dynamics
}

\author{
L. Wang*, J. C. Li, J. F. Zhou \\ Institute of Mechanics, Chinese Academy of Sciences, Beijing 100080, China \\ Email: wanglong@imech.ac.cn
}

\begin{abstract}
Coarse Particle sedimentation is studied by using an algorithm with no adjustable parameters based on stokesian dynamics. Only inter-particle interactions of hydrodynamic force and gravity are considered. The sedimentation of a simple cubic array of spheres is used to verify the computational results. The scaling and parallelism with OpenMP of the method are presented. Random suspension sedimentation is investigated with Mont Carlo simulation. The computational results are shown in good agreement with experimental fitting at the lower computational cost of $O(N \ln N)$.
\end{abstract}

Key words: sedimentation, coarse particle, Stokesian dynamics

\section{INTRODUCTION}

Particle suspension dispersion systems play important roles in sediment transport process, in which particle settling velocity is a crucial parameter. The sedimentation of particles in the real environment is rather complex. Many factors such as particle size distribution, salinity, turbulence etc. may exert effects on sedimentation. As a preliminary study, the sedimentation of coarse particles, which is governed principally by gravity and inter-particle hydrodynamic forces, is considered. Correct evaluation of the hydrodynamic forces involves multibody interactions. Durlofsky [1] developed a method that accounts for both the multibody interactions and the lubrication effects by splitting the hydrodynamic intcraction into a far-ficld mobility and a near-ficld pairwise additive resistance calculation. The method is named as Stokesian Dynamics (SD). The main disadvantage of SD, however, is that it requires inversion of a far-field mobility matrix with a computational cost of order $O\left(N^{3}\right)$, which limits the particle number to the order of hundreds. Sierou[2] put forward Accelerated Stokesian Dynamics (ASD), which reduces the computational cost to $O(N \ln N)$ with the same accuracy as SD by use of iterative method and particle-mesh-Ewald algorithms. However, extra parameter in the Sierou paper is added to assure the positive definiteness of resulting equation. In this paper, an algorithm is presented by restating Sierou's method in more compact matrix form by introducing reflection matrix. With this compact matrix form, the adjustable parameter is unnecessary. Simple-cubic array of spheres sedimentation is calculated and the results are compared with exact solutions. Scaling and parallel efficiency of this method is also shown. Finally, random sedimentation is studied.

\section{METHOD}

With multipole expansion, the disturbance velocity at any point in fluid due to particles may be expressed as

$u_{i}^{\prime}(x)=\frac{1}{8 \pi \mu} \sum_{n}\left(1+\frac{a^{2}}{6} \nabla^{2}\right) J_{i j} F_{j}^{n}+T_{j}^{n} R_{i j}+S_{j k}^{n}\left(1+\frac{a^{2}}{10} \nabla^{2}\right) K_{i j k}+\cdots$

where $u_{i}^{\prime}$ is disturbance velocity, $\mu$ viscosity, a particle radius, $F$ force, $T$ torque, $S$ stress tensor, $J, R, K$ basic solutions of Stokesian equation. Combing equation (1) with the Faxen laws, one may obtain the relationship between the induced moments on the particle $m$ and the moments on particle $n[7]$ 


$$
\left(\begin{array}{l}
F^{m} \\
T^{m} \\
S^{m}
\end{array}\right)=I_{B}\left(\begin{array}{l}
U^{\infty}-U^{m} \\
\Omega^{\infty}-\Omega^{m} \\
E^{\infty}
\end{array}\right)-\sum_{n \neq m} \tilde{R}_{m n}\left(\begin{array}{c}
F^{n} \\
T^{n} \\
S^{n}
\end{array}\right)
$$

where $\tilde{R}_{m n}$ is called reflection matrix, $U^{\infty}, \Omega^{\infty}, E^{\infty}$ respectivcly is far field velocity, angular velocity, and strain tensor. $U^{m}, \Omega^{m}$ is particle $m$ velocity, angular velocity.

To rapidly calculate the moments on particles, a conjugate-gradient-type iterative method, GMRES (generalized minimum residual) method is used. The key step in the above-mentioned calculation is to implement the matrix-vector multiplication of the reflection matrix with moments $F, T, S$. Just as PME method, we distribute the moments on the particle over the mesh. And fast Fourier transform (FFT) is used to calculate the wave-space part of interactions, while the real-part of interaction of interaction can be calculated with the computational cost of $O(N)$ by introduction of a cutoff radius. Since reflection method can not entirely capture the hydrodynamic interactions, lubrication correction in the near field is needed owing to the truncation of multipole expansion [1]. Thus, the particle motion equations can be written as

$$
\left[R_{l u b}+\left(I+\tilde{R}_{f}\right)^{-1} I_{B}\right]\left(\begin{array}{l}
U^{\infty}-U \\
\Omega^{\infty}-\Omega \\
E^{\infty}
\end{array}\right)=\left(\begin{array}{l}
F^{H} \\
T^{H} \\
S^{I I}
\end{array}\right)
$$

where $R_{l u b}$ is lubrication matrix. $\tilde{R}_{f}$ is composed of $\tilde{R}_{m n}$. To solve the cquation, reflection equation (2) is combined, the resultant equation is

$R_{l u b} I_{B}^{-1}\left(I+\tilde{R}_{f}\right)\left(\begin{array}{c}F \\ T \\ S\end{array}\right)+\left(\begin{array}{c}F \\ T \\ S\end{array}\right)=\left(\begin{array}{c}F^{H} \\ T^{H} \\ S^{H}\end{array}\right)$

After substituting the solution back into equation (2), the particle translation and angular vclocity can be obtained. Sierou [2] solved the equation (3) by another form with an adjustable parameter to speed up convergence. We would rather choose hydrodynamic force instead of velocity as direct unknowns. Therefore, no adjustable parameter is needed in the present mothod.

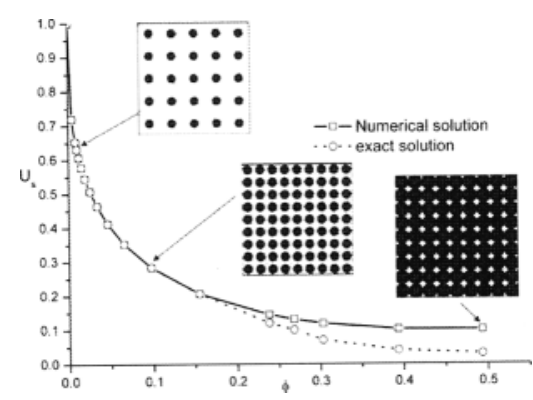

Figure 1: The Sedimentation velocity of simple cubic array vs. different volume fraction

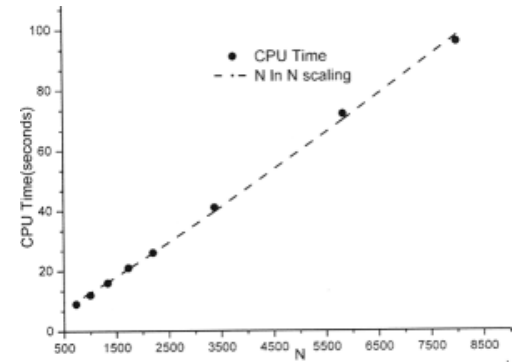

Figure 2: The CPU time $T$ (in seconds) as function of particle number $N$ for convergence accuracy of $10^{-4}$

\section{RESULTS}

To verify the method and program, the sedimentation of simple-cubic arrays is calculated and compared with the exact solution by Zick and Homsy [4]. In Figure 1, non-dimensional sedimentation velocity of a simple cubic array is presented. The cutoff radius for real-space part of far-field interactions is $4 \sqrt{3} a$. Wave-space part of far field interactions is carried out by FFTW. For volume fraction less than $20 \%$, our numerical solution is almost identical to the exact solution. For high volume fraction, numerical solution overestimates the sedimentation velocity, the reason of which is the omission of high-order multipole. For these calculations, iteration less than 10 is enough for convergence 
requirement. In Figure $2 \mathrm{CPU}$ time $T$ in seconds as a function of particle number $N$ is shown for volume fraction of particles $45.2 \%$ with a convergence accuracy of $10^{-4}$. Configurations of the particles are simple cubic arrays. All the runs is performed on Intel Pentium 4, 3.0GHz processor and the elapsed time is given in seconds. In our program, in order to efficiently calculate the real-space part of far-field interactions and near-field lubrication force, the cell list method is applied, which reduces computational cost from $O\left(N^{2}\right)$ to $O(N)$. The actual calculation time accords with the expected $N \ln N$ scaling.

For more efficient calculation, the parallelization is implemented with OpenMP system. In this case, the particle number in simple-cubic configuration is increased to 5832 with grid points $270^{3}$. A linear speedup can be observed in Figure 3.

The sedimentation of random suspensions is also carried out with Monte Carlo simulation, in which gravity is the unique external force. For sedimentation system, Mo and A.S. Sangani [5] gave a corrected sedimentation velocity formula considering the size effect,

$U_{s}=U_{s}(N)+1.7601(\phi / N)^{1 / 3} \mu_{r} S(0) U_{0}$

where, $U_{s}(N)$ is a computational result with $\mathrm{N}$ particles. Richardson and Zaki [6] put forward an empirical formula for coarse particle sedimentation velocity:

$U_{s}=(1-\phi)^{m} U_{0}$

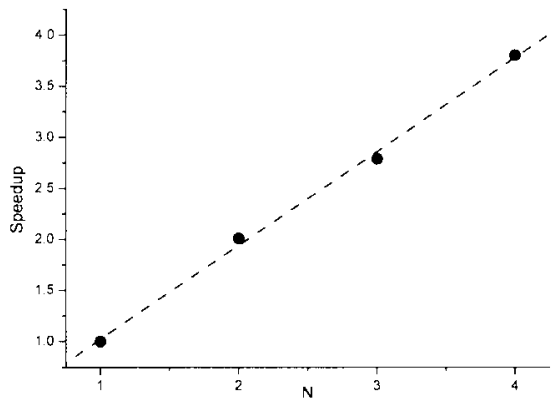

Figure 3: The Speedup of $\mathrm{N}$ processors with OpenMP. The solid circle is speedup.

Fitting is done with the dash line

$m$ is 5.9 in our data fitting. We find in Figure 4 that corrected results and fitting data denoted by solid circle and triangle respectively, are very close to each other. The departure is partially due to the truncation of multipole. Another reason is that the requirement of uniform size in particles in our computation is very hard to meet in the experiment.

\section{CONCLUSIONS}

In this study, the sedimentation velocity of coarse particles is investigated with stokesian dynamies. In order to validate the method, a simple cubic array of spheres is used to calculate the sedimentation velocity dependency on volume fraction. The comparison with exact results is satisfied. The computational cost in the method has proved to been reduced from $O\left(N^{3}\right)$ to $N \ln (N)$. The parallel performances of the method with Open MP show a good linear scaling. Mont Carlo simulations are adopted to investigate random suspension sedimentation. The results agree well with empirical formula. The method used in the present paper is capable of predicting the sedimentation behaviors of suspension dispersion system with large amount of particles. Beside hydrodynamic interactions, further study on flocculation in salty water should take other inter-particle potential force into consideration. 


\section{Acknowledgements}

This study is financially supported by China National Natural Science Foundations (Grant No. 10332050 and 10572144). The authors are grateful to CAS for the support of knowledge innovation program (Grant No. KJCX-CW-L08) and appreciate An Yi for his help in the management of computer system.

\section{REFERENCES}

1. Durlofsky L, Brady JF. Dynamic simulation of hydro-dynamically interacting particles. J. Fluid Mech, 1987;180:21-49

2. Sierou A, Brady JF. Accelerated Stokesian Dynamics simulations. J. Fluid Mech, 2001;448:115146

3. Hasimoto H. On the periodic fundamental solutions of the Stokes equations and their application to viscous flow past a cubic array of spheres. J. Fluid Mech, 1959;5:317-328

4. Zick AA, Homsy GM. Stokes flow through periodic arrays of spheres. J. Fluid Mech, 1982;115:1328

5. Mo G., Sangani AS. A method for computing Stokes flow interactions among spherical objects and its application to suspensions of drops and porous materials. Phys. Fluids, 1994:6:1637-1652

6. Richardson JR, Zaki WN. Sedimentation and fluidization: I. Trans. Inst. Chem. Eng., 1954;32:3553

7. Ichiki K, Brady JF. Many-body effects and matrix inversion in low-Reynolds-number hydrodynamics. Phys. Fluids, 2001;13:350-353 